\title{
A note on weak fuzzy P-spaces
}

Thangaraj $\mathrm{G}^{*} \cdot 1$ and Anbazhagan $\mathrm{C}^{2}$

Received: 20 January 2021/ Accepted: 10 April 2021/ Published online: 18 June 2021

(C)Sacred Heart Research Publications 2017

\begin{abstract}
In this paper, the concepts of weak fuzzy $P$-spaces, are extensively established. The inter-relations of weak fuzzy $P$-spaces, fuzzy $P$-spaces, fuzzy almost $P$-spaces, fuzzy weakly Lindelöf spaces and fuzzy almost Lindelöf spaces are also investigated in this paper.

Key words: Fuzzy dense set, Fuzzy $G_{\delta}$-set, Fuzzy Regular open set, Fuzzy $P$-space, Fuzzy almost $P$-space, weak Fuzzy $P$-space, Fuzzy weakly Lindelöf space, Fuzzy almost Lindelöf space.

AMS classification: 54A40, 03E72.
\end{abstract}

\section{Introduction}

The concept of fuzzy sets and fuzzy set operations were first introduced by L.A.Zadeh in his classical paper [16] in the year 1965. Thereafter the paper of C.L.Chang [5] in1968 paved the way for the subsequent tremendous growth of the numerous fuzzy topological concepts. Since then much attention has been paid to generalize the basic concepts of General Topology in fuzzy setting andthus a modern theory of fuzzy topology has been developed. In recent years, fuzzy topology has been found to be very useful in solving many practical problems. In this paper, the concepts of weak fuzzy $P$-spaces, are extensively established. The inter-relations of weak fuzzy $P$-spaces, fuzzy $P$-spaces, fuzzy almost $P$-spaces, fuzzy weakly Lindelöf spaces and fuzzy almost Lindelöf spaces are also investigated in this paper.

\section{Preliminaries}

Now we give some basic notions and results used in the sequel. In this work by $(X, T)$ or simply by $X$, we will denote a fuzzy topological space due to Chang (1968).

\footnotetext{
${ }^{1}$ Department of Mathematics, Thiruvalluvar University, Vellore - 632115, Tamil Nadu, India.

${ }^{2}$ Department of Mathematics, Jawahar Science College, Neyveli - 607803, Tamil Nadu, India.

Emails: *,1 g.thangaraj@rediffmail.com, ${ }^{2}$ emailofca@gmail.com
} 
ISSN: 2456-8686, 5(1), 2021:038-047

https://doi.org/10.26524/cm90

Let $X$ be a non-empty set and $I$, the unit interval $[0,1]$. A fuzzy set $\lambda$ in $X$ is a function from $X$ into $I$. The null set 0 is the function from $X$ into $I$ which assumes only the value 0 and the whole fuzzy set 1 is the function from $X$ into $I$ which takes 1 only.

Definition 2.1 [5] Let $\lambda$ and $\mu$ be fuzzy sets in $X$. Then for all $x \in X$,

1. $\lambda=\mu \Leftrightarrow \lambda(x)=\mu(x)$,

2. $\lambda \leq \mu \Leftrightarrow \lambda(x) \leq \mu(x)$,

3. $\psi=\lambda \vee \mu \Leftrightarrow \psi(x)=\max \{\lambda(x), \mu(x)\}$,

4. $\delta=\lambda \wedge \mu \Leftrightarrow \delta(x)=\min \{\lambda(x), \mu(x)\}$,

5. $\eta=\lambda^{c} \Leftrightarrow \eta(x)=1-\lambda(x)$.

For a family $\left\{\lambda_{i} / i \in I\right\}$ of fuzzy sets in $X$, the union $\psi=\vee_{i} \lambda_{i}$ and intersection $\delta=\wedge_{i} \lambda_{i}$ are defined by $\psi(x)=\sup _{i}\left\{\lambda_{i}(x), x \in X\right\}$, and $\delta(x)=$ inf $_{i}\left\{\lambda_{i}(x), x \in X\right\}$.

The fuzzy set $0_{X}$ is defined as $0_{X}(x)=0$, for all $x \in X$ and the fuzzy set $1_{X}$ defined as $1_{X}(x)=1$, for all $x \in X$.

Definition 2.2 [6] A fuzzy point $x_{\alpha}$ in $X$ is a fuzzy set with membership function defined as:

$$
x_{\alpha}(y)=\left\{\begin{array}{cc}
\alpha & \text { if } x=y \\
0 & \text { if } x \neq y
\end{array}\right.
$$

Definition 2.3 [5] A fuzzy topology is a family ' $T$ ' of fuzzy sets in $X$ which satisfies the following conditions:

(1) $\Phi, X \in T$,

(2) If $A, B \in T$, then $A \cap B \in T$,

(3) If $A_{i} \in T$, for each $i \in I$, then $\cup_{i \in I} A_{i} \in T$.

$T$ is called a fuzzy topology for $X$ and the pair $(X, T)$ is a fuzzy topological space or fts in short. Every member of $T$ is called a $T$-open fuzzy set. A fuzzy set is $T$-closed if and only if its complement is $T$-open. When no confusion is likely to arise, we shall call a $T$-open ( $T$-closed) fuzzy set simply an open (closed) fuzzy set. 
ISSN: 2456-8686, 5(1), 2021:038-047

https://doi.org/10.26524/cm90

Definition 2.4 [5] Let $(X, T)$ be any fuzzy topological space and $\lambda$ be any fuzzy set in $(X, T)$. The closure and interior of a fuzzy set $\lambda$ in a fuzzy topological space $(X, T)$ are respectively denoted as $\operatorname{cl}(\lambda)$ and $\operatorname{int}(\lambda)$ are defined as

(1) $\operatorname{cl}(\lambda)=\wedge\{\mu / \lambda \leq \mu, 1-\mu \in T\}$ and

(2) $\operatorname{int}(\lambda)=\vee\{\mu / \mu \leq \lambda, \mu \in T\}$.

Lemma 2.5 [15] For a fuzzy set $\lambda$ of a fuzzy space $X$,

(a) $1-\operatorname{cl}(\lambda)=\operatorname{int}(1-\lambda)$ and

(b) $1-\operatorname{int}(\lambda)=\operatorname{cl}(1-\lambda)$.

Lemma 2.6 [1] For a family $\mathcal{A}=\left\{\lambda_{a}\right\}$ of fuzzy sets of a fuzzy space $X, \vee c l \lambda_{\alpha} \leq$ $c l\left(\vee \lambda_{\alpha}\right)$. In case $\mathcal{A}$ is a finite set, $\vee c l\left(\lambda_{\alpha}\right)=\operatorname{cl}\left(\vee \lambda_{\alpha}\right)$. Also $\vee \operatorname{int} \lambda_{\alpha} \leq \operatorname{int}\left(\vee \lambda_{\alpha}\right)$.

Definition 2.7 [2] A fuzzy set $\lambda$ in a fuzzy topological space $(X, T)$ is called a fuzzy $F_{\sigma}$-set in $(X, T)$ if $\lambda=\vee_{i=1}^{\infty}\left(\lambda_{i}\right)$, where $1-\lambda_{i} \in T$ for $i \in I$.

Definition 2.8 2] A fuzzy set $\lambda$ in a fuzzy topological space $(X, T)$ is called a fuzzy $G_{\delta^{-}}$set in $(X, T)$ if $\lambda=\wedge_{i=1}^{\infty}\left(\lambda_{i}\right)$, where $\lambda_{i} \in T$ for $i \in I$.

Definition 2.9 [13] A fuzzy set $\lambda$ in a fuzzy topological space $(X, T)$ is called a fuzzy dense set if there exists no fuzzy closed set $\mu$ in $(X, T)$ such that $\lambda<\mu<1$.

Definition 2.10 [8] A non-zero fuzzy set $\lambda$ in a fuzzy topological space $(X, T)$ is called a fuzzy somewhere dense set if $\operatorname{int} \operatorname{cl}(\lambda) \neq 0$ in $(X, T)$.

Definition 2.11 [14] Let $(X, T)$ be a fuzzy topological space. A fuzzy set $\lambda$ in $(X, T)$ is called a fuzzy $\sigma$-nowhere dense set if $\lambda$ is a fuzzy $F_{\sigma}$-set in $(X, T)$ such that $\operatorname{int}(\lambda)=0$.

Definition 2.12 [11] A fuzzy topological space $(X, T)$ is called a fuzzy $P$-space if countable intersection of fuzzy open sets in $(X, T)$ is fuzzy open. That is, every non-zero fuzzy $G_{\delta}$ set in $(X, T)$ is fuzzy open in $(X, T)$. 
ISSN: 2456-8686, 5(1), 2021:038-047

https://doi.org/10.26524/cm90

Definition 2.13 [12] A fuzzy topological space $(X, T)$ is called a fuzzy almost Lindelöf space if every fuzzy open cover $\left\{\lambda_{\alpha}\right\}_{\alpha \in \Delta}$ of $(X, T)$ admits a countable subcover $\left\{\lambda_{n}\right\}_{n \in N}$ such that $\vee_{n \in N} c l\left(\lambda_{n}\right)=1$.

Definition 2.14 [12] A fuzzy topological space $(X, T)$ is said to be fuzzy weakly Lindelöf if for every fuzzy open cover $\left\{\lambda_{\alpha}\right\}_{\alpha \in \Delta}$ of $(X, T)$ there exists a countable subcover $\left\{\lambda_{n}\right\}_{n \in N}$ such that $\operatorname{cl}\left[\vee_{n \in N}\left(\lambda_{n}\right)\right]=1$.

Definition 2.15 [10] A fuzzy topological space $(X, T)$ is called a fuzzy almost $P$-space if for every non-zero fuzzy $G_{\delta^{-}}$set $\lambda$ in $(X, T)$, int $(\lambda) \neq 0$ in $(X, T)$.

Definition 2.16 [7] A fuzzy topological space $(X, T)$ is called a fuzzy hyperconnected space if every fuzzy open set $\lambda$ is fuzzy dense in $(X, T)$. That is, $\operatorname{cl}(\lambda)=1$ for all $\lambda(\neq 0) \in T$.

Definition 2.17 A fuzzy set $\lambda$ in a fuzzy topological space $X$ is called

(1) fuzzy pre-open if $\lambda \leq \operatorname{intcl}(\lambda)$ and fuzzy pre-closed if $\operatorname{clint}(\lambda) \leq \lambda[4$.

(2) fuzzy semi-open if $\lambda \leq \operatorname{clint}(\lambda)$ and fuzzy semi-closed if $\operatorname{intcl}(\lambda) \leq \lambda[4$.

(3) fuzzy $\beta$-open if $\lambda \leq \operatorname{clintcl}(\lambda)$ and fuzzy $\beta$-closed if $\operatorname{int} \operatorname{clint}(\lambda) \leq \lambda[3$.

(4) fuzzy regular open if $\operatorname{int} \operatorname{cl}(\lambda)=\lambda$ and fuzzy regular closed if $\operatorname{clint}(\lambda)=\lambda[1$.

Theorem 2.18 [1] In a fuzzy topological space $(X, T)$,

(a). The closure of a fuzzy open set is a fuzzy regular closed set

(b). The interior of a fuzzy closed set is a fuzzy regular open set.

Theorem 2.19 [9] A fuzzy topological space $(X, T)$ is a weak fuzzy $P$-space if and only if $\vee_{i=1}^{\infty}\left(\mu_{i}\right)$, where $\left(\mu_{i}\right)$ 's are fuzzy regular closed sets in $(X, T)$, is fuzzy regular closed in $(X, T)$.

Theorem 2.20 [9] If a fuzzy topological space $(X, T)$ is a weak fuzzy $P$-space, then $c l\left(\vee_{i=1}^{\infty}\left(\lambda_{i}\right)\right)=\vee_{i=1}^{\infty}\left(c l\left(\lambda_{i}\right)\right)$, where $\left(\lambda_{i}\right)$ 's are non-zero fuzzy open sets in $(X, T)$. 


\section{Weak fuzzy P-spaces}

Definition 3.1 [9] A fuzzy topological space $(X, T)$ is called a weak fuzzy $P$-space if the countable intersection of fuzzy regular open sets in $(X, T)$ is a fuzzy regular open set in $(X, T)$. That is., $\wedge_{i=1}^{\infty}\left(\lambda_{i}\right)$ is fuzzy regular open in $(X, T)$, where $\left(\lambda_{i}\right)^{\prime}$ s are fuzzy regular open sets in $(X, T)$.

\section{Characterizations of weak fuzzy $P$-spaces}

Proposition 4.1 If $\lambda=\vee_{i=1}^{\infty}\left(\operatorname{cl}\left(\lambda_{i}\right)\right)$, where $\left(\lambda_{i}\right)$ 's are fuzzy open sets in a weak fuzzy $P$-space $(X, T)$ and $\lambda \neq 1$, then $\lambda$ is not a fuzzy dense set in $(X, T)$.

Proof: Suppose that $\lambda=\vee_{i=1}^{\infty}\left[\operatorname{cl}\left(\lambda_{i}\right)\right]$, where $\left(\lambda_{i}\right)^{\prime}$ 's are fuzzy open sets in a weak fuzzy $P$-space $(X, T)$ and $\lambda \neq 1$. Now, $\left(\lambda_{i}\right)$ 's are fuzzy open sets in $(X, T)$, implies that $\left(c l\left(\lambda_{i}\right)\right)$ 's are fuzzy regular closed sets in $(X, T)$. Since $(X, T)$ is a weak fuzzy $P$-space, by theorem (2.19), $\vee_{i=1}^{\infty}\left[c l\left(\lambda_{i}\right)\right]$ is a fuzzy regular closed set in $(X, T)$ and hence $\lambda$ is a fuzzy regular closed set in $(X, T)$. Then, $\operatorname{clint}(\lambda)=\lambda$, in $(X, T)$. It has to be proved that $\operatorname{cl}(\lambda) \neq 1$, in $(X, T)$. Assume the contrary. Suppose that $\operatorname{cl}(\lambda)=1$, in $(X, T)$. Then, $\operatorname{cl}[\operatorname{clint}(\lambda)]=\operatorname{cl}(\lambda)=1$, in $(X, T)$ and hence $\operatorname{clint}(\lambda)=1$, in $(X, T)$. Then, $\operatorname{clint}(\lambda)=1$ and $\operatorname{clint}(\lambda)=\lambda$, implies that $\lambda=1$, contradiction. Hence, the assumption that $\operatorname{cl}(\lambda)=1$, does not hold in $(X, T)$ and therefore, $\operatorname{cl}(\lambda) \neq 1$, in $(X, T)$. Thus, $\lambda$ is not a fuzzy dense set in $(X, T)$.

Proposition 4.2 If $\lambda=\vee_{i=1}^{\infty}\left(\operatorname{cl}\left(\lambda_{i}\right)\right)$, where $\left(\lambda_{i}\right)$ 's are fuzzy open sets in a weak fuzzy $P$-space $(X, T)$ and $\lambda \neq 1$, then $1-\lambda$ is a fuzzy somewhere dense set in $(X, T)$.

Proof: Suppose that $\lambda=\vee_{i=1}^{\infty}\left[c l\left(\lambda_{i}\right)\right]$, where $\left(\lambda_{i}\right) \in T$. Now, $\left(\lambda_{i}\right)$ 's are fuzzy open sets in $(X, T)$, implies that $\left(c l\left(\lambda_{i}\right)\right)$ 's are fuzzy regular closed sets in $(X, T)$. Since $(X, T)$ is a weak fuzzy $P$-space, by theorem 2.19$), \vee_{i=1}^{\infty}\left[\operatorname{cl}\left(\lambda_{i}\right)\right]$ is a fuzzy regular closed set in $(X, T)$ and hence $\lambda$ is a fuzzy regular closed set in $(X, T)$. Then, $\operatorname{clint}(\lambda)=\lambda$, in $(X, T)$. This implies that $1-\operatorname{clint}(\lambda)=1-\lambda \neq 0$, in $(X, T)$ and thus intcl $(1-\lambda)=1-\lambda \neq 0$, in $(X, T)$. Therefore, $1-\lambda$ is a fuzzy somewhere dense set in $(X, T)$.

Proposition 4.3 If $\lambda=\vee_{i=1}^{\infty}\left(\operatorname{cl}\left(\lambda_{i}\right)\right)$, where $\left(\lambda_{i}\right)$ 's are fuzzy open sets in a weak fuzzy $P$-space $(X, T)$ and $\lambda \neq 1$, then $\operatorname{int}\left(\vee_{i=1}^{\infty}\left(c l\left(\lambda_{i}\right)\right)\right) \neq 0$, in $(X, T)$. 
Proof: Suppose that $\lambda=\vee_{i=1}^{\infty}\left[\operatorname{cl}\left(\lambda_{i}\right)\right]$, where $\left(\lambda_{i}\right) \in T$ and $\lambda \neq 1$. Now, $\left(\lambda_{i}\right)^{\prime}$ 's are fuzzy open sets in $(X, T)$, implies that $\left(c l\left(\lambda_{i}\right)\right)$ 's are fuzzy regular closed sets in $(X, T)$. Since $(X, T)$ is a weak fuzzy $P$-space, by theorem 2.19$), \vee_{i=1}^{\infty}\left[\operatorname{cl}\left(\lambda_{i}\right)\right]$ is a fuzzy regular closed set in $(X, T)$ and hence $\lambda$ is a fuzzy regular closed set in $(X, T)$. Then, $\operatorname{clint}(\lambda)=\lambda$, in $(X, T)$. This implies that $\operatorname{int}(\lambda) \neq 0$, in $(X, T) . \quad[$ For, if $\operatorname{int}(\lambda)=0$ in $(X, T)$ will imply that $\operatorname{clint}(\lambda)=\operatorname{cl}(0)=0 \neq \lambda$ in $(X, T)$, a contradiction]. Thus, $\operatorname{int}\left(\vee_{i=1}^{\infty}\left(\operatorname{cl}\left(\lambda_{i}\right)\right)\right) \neq 0$, where $\left(\lambda_{i}\right)$ 's are fuzzy open sets in $(X, T)$.

Proposition 4.4 If $\lambda=\vee_{i=1}^{\infty}\left(c l\left(\lambda_{i}\right)\right)$, where $\left(\lambda_{i}\right)$ 's are fuzzy open sets in a weak fuzzy $P$-space $(X, T)$ and $\lambda \neq 1$, then $\lambda$ is not a fuzzy $\sigma$-nowhere dense set in $(X, T)$.

Proof: Suppose that $\lambda=\vee_{i=1}^{\infty}\left[\operatorname{cl}\left(\lambda_{i}\right)\right]$, where $\left(\lambda_{i}\right) \in T$. Now, $\left(\lambda_{i}\right)^{\prime}$ 's are fuzzy open sets in $(X, T)$, implies that $\left(c l\left(\lambda_{i}\right)\right)$ 's are fuzzy regular closed sets in $(X, T)$. Also, since fuzzy regular closed sets are fuzzy closed sets in a fuzzy topological space, $\lambda=\vee_{i=1}^{\infty}\left[\operatorname{cl}\left(\lambda_{i}\right)\right]$, implies that $\lambda$ is a fuzzy $F_{\sigma}$-set in $(X, T)$. Since $(X, T)$ is a weak fuzzy $P$-space, by theorem (2.19), $\vee_{i=1}^{\infty}\left[c l\left(\lambda_{i}\right)\right]$ is a fuzzy regular closed set in $(X, T)$ and hence $\lambda$ is a fuzzy regular closed set in $(X, T)$. Then, $\operatorname{clint}(\lambda)=\lambda$, in $(X, T)$. This implies that $\operatorname{int}(\lambda) \neq 0$, in $(X, T)$. Thus, $\lambda$ is a fuzzy $F_{\sigma}$-set in $(X, T)$ such that $\operatorname{int}(\lambda) \neq 0$ in $(X, T)$, implies that $\lambda$ is not a fuzzy $\sigma$-nowhere dense set in $(X, T)$.

Proposition 4.5 If $\mu=\wedge_{i=1}^{\infty}\left(\operatorname{int}\left(\mu_{i}\right)\right)$, where $\left(\mu_{i}\right)$ 's are fuzzy closed sets in a weak fuzzy $P$-space $(X, T)$, then $\mu$ is not a fuzzy dense set in $(X, T)$ and $c l\left(\wedge_{i=1}^{\infty}\left(\operatorname{int}\left(\mu_{i}\right)\right)\right) \neq 0$, in $(X, T)$.

Proof: Suppose that $\mu=\wedge_{i=1}^{\infty}\left(\operatorname{int}\left(\mu_{i}\right)\right)$, where $\left(\mu_{i}\right)$ 's are fuzzy closed sets in a weak fuzzy $P$-space $(X, T)$. Now, $\left(\mu_{i}\right)$ 's are fuzzy closed sets in $(X, T)$, implies that $\left(\operatorname{int}\left(\mu_{i}\right)\right)$ 's are fuzzy regular open sets in $(X, T)$ and hence $\left[1-\left(\operatorname{int}\left(\mu_{i}\right)\right)\right]$ 's are fuzzy regular closed sets in $(X, T)$. Since $(X, T)$ is a weak fuzzy $P$-space, by theorem (2.19), $\vee_{i=1}^{\infty}\left[1-\left(\operatorname{int}\left(\mu_{i}\right)\right)\right]$ is a fuzzy regular closed set in $(X, T)$ and hence $1-\left[\wedge_{i=1}^{\infty}\left(\operatorname{int}\left(\mu_{i}\right)\right)\right]$ is a fuzzy regular closed set in $(X, T)$. Thus, $\wedge_{i=1}^{\infty}\left(\operatorname{int}\left(\mu_{i}\right)\right)$ is a fuzzy regular open set in $(X, T)$ and hence $\mu$ is a fuzzy regular open set in $(X, T)$. This implies that $\operatorname{intcl}(\mu)=\mu$, in $(X, T)$. Hence $\operatorname{cl}(\mu) \neq 1$, in $(X, T)$. [For, if $\operatorname{cl}(\mu)=1$ in $(X, T)$, then $\operatorname{intcl}(\mu)=\operatorname{int}(1)=1 \neq \mu$ in $(X, T)$, a contradiction]. Therefore, $\mu$ is not a fuzzy dense set in $(X, T)$. Also, $\operatorname{cl}(\mu) \neq 0$, in $(X, T)$. [For, if $c l(\mu)=0$ in $(X, T)$, then $\operatorname{intcl}(\mu)=\operatorname{int}(0)=0 \neq \mu$ in $(X, T)$, a contradiction]. Thus, $c l\left(\wedge_{i=1}^{\infty}\left(\operatorname{int}\left(\mu_{i}\right)\right)\right) \neq 0$, in $(X, T)$. 


\section{Weak fuzzy $P$-spaces and other fuzzy topological spaces}

Proposition 5.1 If each fuzzy open set is a fuzzy regular open set in a weak fuzzy $P$-space $(X, T)$, then $(X, T)$ is a fuzzy $P$-space.

Proof: Let $\lambda$ be a fuzzy $G_{\delta}$-set in $(X, T)$. Then, $\lambda=\wedge_{i=1}^{\infty}\left(\lambda_{i}\right)$, where $\left(\lambda_{i}\right)^{\prime}$ 's are fuzzy open sets in $(X, T)$. By hypothesis, each fuzzy open set is a fuzzy regular open set in $(X, T)$. Then, $\left(\lambda_{i}\right)$ 's are fuzzy regular open sets in $(X, T)$. Since $(X, T)$ is a weak fuzzy $P$-space, $\wedge_{i=1}^{\infty}\left(\lambda_{i}\right)$ is a fuzzy regular open set in $(X, T)$ and hence $\lambda$ is a fuzzy regular open set in $(X, T)$. Now, each fuzzy regular open set is a fuzzy open set in a fuzzy topological space $(X, T), \lambda$ is a fuzzy open set in $(X, T)$. Hence, each fuzzy $G_{\delta^{-}}$set in $(X, T)$ is fuzzy open in $(X, T)$, implies that $(X, T)$ is a fuzzy $P$-space.

Proposition 5.2 If each fuzzy closed set is a fuzzy regular closed set in a weak fuzzy $P$-space $(X, T)$, then $(X, T)$ is a fuzzy $P$-space.

Proof: Let $\lambda$ be a fuzzy closed set in $(X, T)$ such that $\operatorname{clint}(\lambda)=\lambda$, in $(X, T)$. Then, $1-\lambda$ is a fuzzy open set in $(X, T)$ such that $1-\operatorname{clint}(\lambda)=1-\lambda$, in $(X, T)$ and hence $\operatorname{intcl}(1-\lambda)=1-\lambda$, in $(X, T)$. Thus, the fuzzy open set $1-\lambda$ is a fuzzy regular open set in the weak fuzzy $P$-space. Hence, by proposition (5.1), $(X, T)$ is a fuzzy $P$-space.

Remark 5.3 Obviously every fuzzy almost Lindelöf space is a fuzzy weakly Lindelöf space. For, $\vee_{n \in N} c l\left(\lambda_{n}\right) \leq c l\left[\vee_{n \in N}\left(\lambda_{n}\right)\right]$ and $\vee_{n \in N} c l\left(\lambda_{n}\right)=1$, implies that $c l\left[\vee_{n \in N}\left(\lambda_{n}\right)\right]=1$.

Proposition 5.4 If the fuzzy topological space $(X, T)$ is a weak fuzzy $P$-space, then every fuzzy weakly Lindelöf space is a fuzzy almost Lindelöf space.

Proof: Let $(X, T)$ be a fuzzy weakly Lindelöf space and $\left\{\lambda_{\alpha}\right\}_{\alpha \in \Delta}$ be a fuzzy open cover of $(X, T)$. Then there exists a countable fuzzy subcover $\left\{\lambda_{n}\right\}_{n \in N}$ such that $c l\left[\vee_{n \in N}\left(\lambda_{n}\right)\right]=1$, in $(X, T)$. Since $(X, T)$ is a weak fuzzy $P$-space, by theorem (2.20), $\operatorname{cl}\left[\mathrm{\vee}_{n \in N}\left(\lambda_{n}\right)\right]=\vee_{n \in N} \operatorname{cl}\left(\lambda_{n}\right)$ where $\left(\lambda_{n}\right)$ 's are non-zero fuzzy open sets in $(X, T)$. Hence, for the fuzzy open cover $\left\{\lambda_{\alpha}\right\}_{\alpha \in \Delta}$ of $(X, T)$, there exists a countable subcover $\left\{\lambda_{n}\right\}_{n \in N}$ such that $\vee_{n \in N} c l\left(\lambda_{n}\right)=1$. Therefore $(X, T)$ is a fuzzy almost Lindelöf space. 
ISSN: 2456-8686, 5(1), 2021:038-047

https://doi.org/10.26524/cm90

Example 5.5 Let $X=\{a, b, c\}$. The fuzzy sets $\lambda, \mu$ and $\nu$ are defined on $X$ as follows:

$\lambda: X \rightarrow[0,1]$ is defined as $\lambda(a)=0.5 ; \quad \lambda(b)=0.6 ; \quad \lambda(c)=0.4$

$\mu: X \rightarrow[0,1]$ is defined as $\mu(a)=0.4 ; \mu(b)=0.7 ; \quad \mu(c)=0.5$

$\nu: X \rightarrow[0,1]$ is defined as $\nu(a)=0.5 ; \quad \nu(b)=0.8 ; \quad \nu(c)=0.6$.

Let $T=\{0, \lambda, \mu, \nu, \lambda \vee \mu, \lambda \wedge \mu, 1\}$ is a fuzzy topology on $X$. Now for the fuzzy $G_{\delta^{-}}$sets $\lambda \wedge \mu \wedge \nu$ and $\{(\lambda \vee \mu) \wedge(\lambda \wedge \mu)\}$ in $(X, T)$, int $(\lambda \wedge \mu \wedge \nu)=\lambda \wedge \mu \neq 0$ and $\operatorname{int}[(\lambda \vee \mu) \wedge(\lambda \wedge \mu)]=\lambda \wedge \mu \neq 0$, in $(X, T)$. Hence $(X, T)$ is a fuzzy almost $P$-space.

Remark 5.6 (1) Clearly every fuzzy $P$-space is a fuzzy almost $P$-space, since for every non-zero fuzzy $G_{\delta}$-set $\delta$ in $(X, T)$, int $(\delta)=\delta \neq 0$. But the converse need not be true. For, in example (5.5), for every non-zero fuzzy $G_{\delta^{-}}$set $\delta$ in $(X, T)$, $\operatorname{int}(\delta) \neq 0$ in $(X, T)$. Hence $(X, T)$ is a fuzzy almost $P$-space, but $(X, T)$ is not a fuzzy $P$-space, since the fuzzy $G_{\delta}$-set $\{(\lambda \vee \mu) \wedge(\lambda \vee \nu) \wedge(\mu \vee \nu)\}$ is not a fuzzy open set in $(X, T)$.

(2) A fuzzy almost $P$-space need not be a weak fuzzy $P$-space. For, consider the following example:

Example 5.7 Let $X=\{a, b, c\}$. The fuzzy sets $\lambda, \mu$ and $\nu$ are defined on $X$ as follows:

$\lambda: X \rightarrow[0,1]$ is defined as $\lambda(a)=0.8 ; \quad \lambda(b)=0.6 ; \quad \lambda(c)=0.7$

$\mu: X \rightarrow[0,1]$ is defined as $\mu(a)=0.6 ; \mu(b)=0.9 ; \quad \mu(c)=0.8$

$\nu: X \rightarrow[0,1]$ is defined as $\nu(a)=0.7 ; \quad \nu(b)=0.5 ; \quad \nu(c)=0.9$.

Let $T=\{0, \lambda, \mu, \nu, \lambda \vee \mu, \lambda \vee \nu, \mu \vee \nu, \lambda \wedge \mu, \lambda \wedge \nu, \mu \wedge \nu, \lambda \wedge(\mu \vee \nu), \lambda \vee(\mu \wedge$ $\nu), \mu \wedge(\lambda \vee \nu), \mu \vee(\lambda \wedge \nu), \nu \wedge(\lambda \vee \mu), \nu \vee(\lambda \wedge \mu), \lambda \wedge \mu \wedge \nu, \lambda \vee \mu \vee \nu, 1\}$ is a fuzzy topology on $X$. In $(X, T)$, for every non-zero fuzzy $G_{\delta}$-set $\delta$, int $(\delta) \neq 0$ in $(X, T)$. Hence $(X, T)$ is a fuzzy almost $P$-space, but $(X, T)$ is not a weak fuzzy $P$-space.

(3) A weak fuzzy $P$-space need not be a fuzzy almost $P$-space. For, Consider the following example:

Example 5.8 Let $X=\{a, b, c\}$. The fuzzy sets $\lambda, \mu$ and $\nu$ are defined on $X$ as follows:

$\lambda: X \rightarrow[0,1]$ is defined as $\lambda(a)=0 ; \quad \lambda(b)=0.4 ; \quad \lambda(c)=0.5$ 
$\mu: X \rightarrow[0,1]$ is defined as $\mu(a)=0.6 ; \quad \mu(b)=0 ; \quad \mu(c)=0.4$

$\nu: X \rightarrow[0,1]$ is defined as $\nu(a)=0.5 ; \quad \nu(b)=0.6 ; \quad \nu(c)=0$.

Let $T=\{0, \lambda, \mu, \nu, \lambda \vee \mu, \lambda \vee \nu, \mu \vee \nu, \lambda \wedge \mu, \lambda \wedge \nu, \mu \wedge \nu, \lambda \wedge(\mu \vee \nu), \lambda \vee(\mu \wedge \nu), \mu \wedge(\lambda \vee$ $\nu), \mu \vee(\lambda \wedge \nu), \nu \wedge(\lambda \vee \mu), \nu \vee(\lambda \wedge \mu), \lambda \wedge \mu \wedge \nu, \lambda \vee \mu \vee \nu, 1\}$ is a fuzzy topology on $X$. Now $\lambda \vee \mu, \lambda \vee(\mu \wedge \nu)$ and $\lambda \vee \mu \vee \nu$ are fuzzy regular open sets in $(X, T)$ and $\{[\lambda \vee \mu] \wedge[\lambda \vee(\mu \wedge \nu)] \wedge[\lambda \vee \mu \vee \nu]=[\lambda \vee(\mu \wedge \nu)]$ is a fuzzy regular open set $(X, T)$ and hence $(X, T)$ is a weak fuzzy $P$-space. But, $(X, T)$ is not a fuzzy almost $P$-space, since for the non-zero fuzzy $G_{\delta}$-set $\{[\lambda \wedge(\mu \vee \nu)] \wedge[\mu \wedge(\lambda \vee \nu)] \wedge[\nu \wedge(\lambda \vee \mu)]\}$, $\operatorname{int}([\lambda \wedge(\mu \vee \nu)] \wedge[\mu \wedge(\lambda \vee \nu)] \wedge[\nu \wedge(\lambda \vee \mu)])=0$, in $(X, T)$.

Proposition 5.9 If a fuzzy topological space $(X, T)$ is a weak fuzzy $P$-space, then $(X, T)$ is not a fuzzy hyperconnected space.

Proof: Let $\mu=\wedge_{i=1}^{\infty}\left(\mu_{i}\right)$, where $\left(\mu_{i}\right)$ 's $(\neq 1)$ are fuzzy regular open sets in $(X, T)$. Since $(X, T)$ is a weak fuzzy $P$-space, $\mu=\wedge_{i=1}^{\infty}\left(\mu_{i}\right)$ is a fuzzy regular open set in $(X, T)$. Since every fuzzy regular open set is a fuzzy open set in a fuzzy topological space $(X, T), \mu$ is a fuzzy open set in $(X, T)$. It has to be proved that $\operatorname{cl}(\mu) \neq 1$ in $(X, T)$. Assume the contrary. Suppose that $c l(\mu)=1$ in $(X, T)$. Then, $c l\left(\wedge_{i=1}^{\infty}\left(\mu_{i}\right)\right)=$ 1 in $(X, T)$. Since $(X, T)$ is a weak fuzzy $P$-space, by theorem $2.20, c l\left(\wedge_{i=1}^{\infty}\left(\mu_{i}\right)\right)=$ $\wedge_{i=1}^{\infty}\left(c l\left(\mu_{i}\right)\right)$ in $(X, T)$. Then, $\wedge_{i=1}^{\infty}\left(c l\left(\mu_{i}\right)\right)=1$ and $c l\left(\mu_{i}\right)=1$, for all $i$. But, $\mu_{i}$ is a fuzzy regular open set in $(X, T)$, implies that $\operatorname{intcl}\left(\mu_{i}\right)=\mu_{i}$ in $(X, T)$ and thus $\operatorname{int}(1)=\mu_{i}$ in $(X, T)$ and hence it will be $\mu_{i}=1$, a contradiction. Hence the assumption $\operatorname{cl}(\mu)=1$ does not hold in $(X, T)$. Thus, $\operatorname{cl}(\mu) \neq 1$ in $(X, T)$, for the fuzzy open set $\mu$ in $(X, T)$, implies that $(X, T)$ is not a fuzzy hyperconnected space.

\section{Conclusions}

In this paper, the concepts of weak fuzzy $P$-spaces, were extensively established and also inter-relations between weak fuzzy $P$-spaces and other fuzzy topological spaces, were investigated.

\section{References}

[1] Azad KK. On fuzzy semicontinuity, fuzzy almost continuity and fuzzy weakly continuity, J. Math. Anal. Appl., 82, 14-32 (1981).

[2] Balasubramanian G, Maximal fuzzy topologies, Kybernetika, 31(5), 459-464 (1995). 
ISSN: 2456-8686, 5(1), 2021:038-047

https://doi.org/10.26524/cm90

[3] Balasubramanian G, Fuzzy $\beta$-open sets and fuzzy $\beta$-separation axiams, Kybernetika, 35(2), 215-223 (1999).

[4] Bin Shahna AS, On fuzzy strong semicontinuity and fuzzy precontinuity, Fuzzy Sets and Systems, 44, 303-308 (1991).

[5] Chang CL, Fuzzy topological spaces, J. Math. Anal. Appl. 24, 182-190 (1968).

[6] Levy R, Almost P-spaces, Canad. J. Math., XXIX(2), 284-288 (1977).

[7] Miguel Caldas, Govindappa Navalagi and Ratnesh Saraf, On fuzzy weakly semiopen functions, Proyecciones, Universidad Católica del Norte, Antofagasta - Chile, 21(1), 51-63 (2002).

[8] Thangaraj G, Resolvability and irresolvability in fuzzy topological spaces, News Bull. Cal. Math. Soc., 3l(4-6), 11-14 (2008).

[9] Thangaraj G, Anbazhagan C and Vivakanandan P, On fuzzy P-spaces, weak fuzzy P-spaces and fuzzy almost P-spaces, Gen. Math. Notes, 18(2), 128-139 (2013).

[10] Thangaraj G and Anbazhagan C, On fuzzy almost P-spaces, International Journal of Innovative Science, Engineering \& Technology, 2(4), 389-407, (2015).

[11] Thangaraj G and Balasubramanian G, On fuzzy basically disconnected spaces, J. Fuzzy Math., 9(1), 103-110 (2001).

[12] Thangaraj G and Balasubramanian G, On some generalizations of fuzzy Lindelöf spaces, J. Fuzzy Math., 15(3), 505-511 (2001).

[13] Thangaraj G and Balasubramanian G, On somewhat fuzzy continuous functions, J. Fuzzy Math., 11(2), 725-736 (2003).

[14] Thangaraj G and Poongothai E, On fuzzy $\sigma$-Baire spaces, Int. J. Fuzzy Math. Sys., 3(4), 275-283 (2013).

[15] Warren RH, Neighborhoods bases and continuity in fuzzy topological spaces, Rocky Mountain J. Math., 8(3), 459-470 (1978).

[16] Zadeh LA, Fuzzy sets, Information and Control, 8, 338-353 (1965). 